(c) ESO 2001

\title{
XMM-Newton observations of the BL Lac MS 0737+7441^
}

\author{
Th. Boller ${ }^{1}$, M. Gliozzi ${ }^{1}$, G. Griffiths ${ }^{2}$, S. Sembay ${ }^{2}$, R. Keil ${ }^{1}$, O. Schwentker ${ }^{1}$, \\ W. Brinkmann ${ }^{1}$, and S. Vercellone ${ }^{3}$ \\ 1 Max-Planck-Institut für extraterrestrische Physik, Postfach 1603, 85748 Garching, Germany \\ 2 X-Ray Astronomy Group, Department of Physics and Astronomy, Leicester University, Leicester LE1 7RH, UK \\ 3 Istituto di Fisica Cosmica "G. Occhialini", CNR, Milano, Italy
}

Received 2 October 2000 / Accepted 26 October 2000

\begin{abstract}
We report on the XMM-Newton observations of the BL Lac object MS 0737.9+7441 during the performance verification phase. A simple power-law fit provides an adequate description of the integrated spectrum in the $0.2-10 \mathrm{keV}$ energy band. The photon index is slightly steeper in the EPIC pn data with $\Gamma=(2.38 \pm 0.01)$ compared to the EPIC MOS data $(\Gamma=(2.28 \pm 0.01))$. The difference is most probably due to the present uncertainties in the calibration of the EPIC MOS and EPIC pn data sets. We report evidence for intrinsic absorption in the distant BL Lac above the Galactic column $\left(N_{\mathrm{H}, \mathrm{Gal}}=3.210^{20} \mathrm{~cm}^{-2}\right)$ which is $N_{\mathrm{H}, \mathrm{fit}}^{z=0.315}=(2.70 \pm 0.20) 10^{20} \mathrm{~cm}^{-2}$ in the EPIC pn data and $N_{\mathrm{H} \text {,fit }}^{z=0.315}=(3.25 \pm 0.25) 10^{20} \mathrm{~cm}^{-2}$ in the EPIC MOS data assuming neutral gas and solar abundances. The flux variations are found to be of the order of $10 \%$. No significant spectral variability is detected.
\end{abstract}

Key words. galaxies: active - galaxies: individual: MS 0737.9+7441 - X-rays: galaxies

\section{Introduction}

The BL Lac object MS 0737.9+7441 was discovered in the Einstein Observatory Extended Medium-Sensitivity Survey (EMSS; Gioia et al. 1990; Stocke et al. 1991) with a flux of $f_{\mathrm{X}}=(9.8 \pm 0.6) 10^{-12} \mathrm{erg} \mathrm{cm}^{-2} \mathrm{~s}^{-1}$ in the energy range between 0.3 and $3.5 \mathrm{keV}$. Its redshift is $z=0.315$ (Morris et al. 1991). In the ROSAT All-Sky-Survey observations the source had a count rate of 0.49 counts $\mathrm{s}^{-1}$ during an exposure of $456 \mathrm{~s}$. Perlman et al. (1996) obtained a best fit power-law photon index of $\Gamma=1.91$ from the observed hardness ratios assuming Galactic absorption $N_{\mathrm{H}, \mathrm{Gal}}=3.210^{20} \mathrm{~cm}^{-2}$. Lamer et al. (1996) examined pointed ROSAT-PSPC data of MS $0737.9+7441$ with a count rate of $0.51 \pm 0.01$ counts per second for an exposure of $8782 \mathrm{~s}$. Using a power-law model the best fit parameters are $N_{\mathrm{H}, \mathrm{fit}}=(4.16 \pm 0.48) 10^{20} \mathrm{~cm}^{-2}$ and $\Gamma=2.39 \pm$ 0.11. In a BeppoSAX observation (Wolter et al. 1998) MS 0737.9+7441 was detected in the LECS instrument with $37.1 \pm 7.8$ net counts during an exposure of $3075 \mathrm{~s}$, the MECS detector net counts were $735.9 \pm 30.6$ in $23279 \mathrm{~s}$. By assuming a simple power law the best fit parameters are $\Gamma=2.53_{-0.23}^{+0.28}$ and $N_{\mathrm{H}, \mathrm{fit}}=\left(25.8_{-21.6}^{+49.3}\right) 10^{20} \mathrm{~cm}^{-2}$.

Send offprint requests to: Th. Boller,

e-mail: bol@mpe.mpg.de

* Based on observations with XMM-Newton, an ESA Science Mission with instruments and contributions directly funded by ESA Member States and the USA (NASA).
A broken power law resulted in photon indices of $\Gamma_{1}=1.17$ (which they classify as uncertain) and $\Gamma_{2}=2.43_{-0.16}^{+0.18}$. The break energy is $1.05(1.27-1.61) \mathrm{keV}$.

In the following we report on the XMM-Newton observation of MS 0737.9+7441 obtained during the performance verification program. Two exposures of approximately $20 \mathrm{ksec}$ and $60 \mathrm{ksec}$ were performed on the source between April 12, 2000 and April 13, 2000, either side of the orbital apogee gap. Around the middle of the second PN exposure the camera suffered a short telemetry break. The PN camera was in full frame mode throughout the observation. Both MOS cameras were in large window $(300 \times 300$ pixels $)$ mode. All cameras employed their respective thin -1 filters.

The Hubble parameter was chosen to be $H_{0}=$ $70 \mathrm{~km} \mathrm{~s}^{-1} \mathrm{Mpc}^{-1}$ and a cosmological deceleration parameter of $q_{0}=\frac{1}{2}$ have been adopted throughout the paper.

\section{Spectral fitting results}

\subsection{EPIC pn results}

The spectral fitting results described below were obtained from the merged data set. Only single events were used from an extraction radius of 75 arcsec around the source position, the background was taken from the same chip with an extraction radius of 110 arcsec. A simple power law fit with the Galactic column fixed to $N_{\mathrm{H}, \mathrm{Gal}}=3.210^{20} \mathrm{~cm}^{-2}$ and allowing the intrinsic 


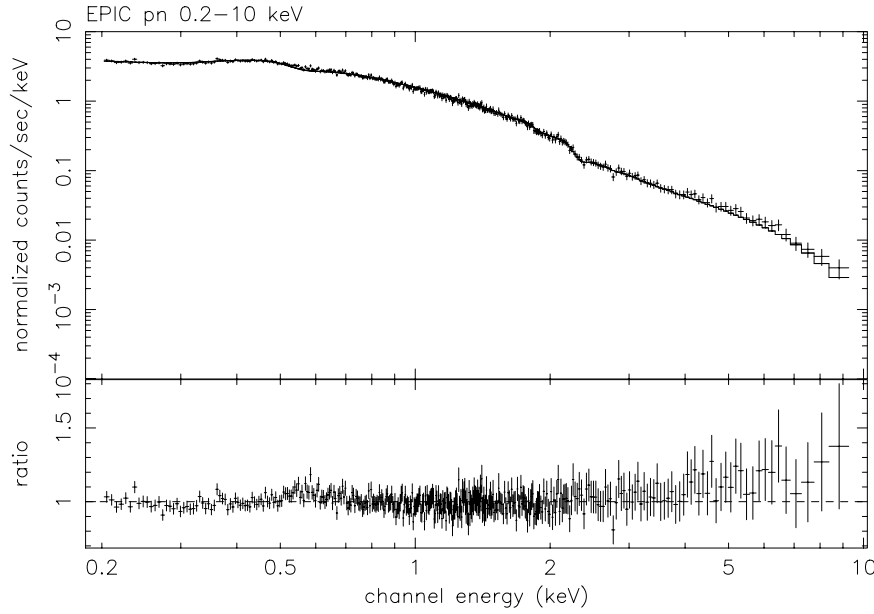

Fig. 1. Power-law fit to the EPIC-pn data. The upper panel shows the count rate distribution of MS 0737.9+7441 (marked by the crosses) in the EPIC pn detector versus energy. The solid line gives the model spectrum. The lower panel gives the ratio between the data and the model

absorption and the photon index to be free parameters provides an adequate fit to the XMM-Newton data $\left(\chi^{2}=375\right.$ for 380 d.o.f.; cf. Fig. 1). We have used the XSPEC models zphabs to access the intrinsic absorption in the source and phabs for the Galactic absorbing column density. The intrinsic absorbing column density measured with the EPIC pn is $N_{\mathrm{H}, \mathrm{fit}}^{z=0.315}=(2.70 \pm 0.2) 10^{20} \mathrm{~cm}^{-2}$ assuming neutral gas and solar abundances, consistent with the excess absorption suggested by the ROSAT data. The photon index is $\Gamma=2.38 \pm 0.01$. The errors correspond to $90 \%$ confidence levels for 1 interesting parameter. Using the $\mathrm{F}$-test for the addition of one free parameter one gets $\Delta \chi^{2} / \chi_{\nu}^{2}=146$ (cf. Eq. (11.50) of Bevington \& Robinson 1992). According to Table C.5 of Bevington \& Robinson (1992) this corresponds to a highly significant improvement (>99.99 per cent) of the fit quality with intrinsic absorption compared to the fit with no intrinsic absorption. The mean $0.2-10 \mathrm{keV}$ absorbed flux obtained from the XMM-Newton observations is $f=8.610^{-12} \mathrm{erg} \mathrm{cm}^{-2} \mathrm{~s}^{-1}$. The unabsorbed flux is $f=1.310^{-11} \mathrm{erg} \mathrm{cm}^{-2} \mathrm{~s}^{-1}$, corresponding to an isotropic luminosity of $L_{\mathrm{X}}=3.610^{45} \mathrm{erg} \mathrm{s}^{-1}$. Spectral residua appear between about 0.5 and $0.7 \mathrm{keV}$ in the EPIC pn spectrum (cf. Fig. 1). These wiggles might be attributed to uncertainties in the presently available EPIC pn response matrix not allowing us to make definitive statements on the possible presence of soft X-ray emission lines, e.g. emission due to O VIII $(653 \mathrm{eV})$ and Fe XXVII $(726 \mathrm{eV})$ would fall in the energy range of the spectral residua. Better calibration and/or deeper observations are needed to further settle this issue. We note that these features do not significantly influence the spectral continuum shape.

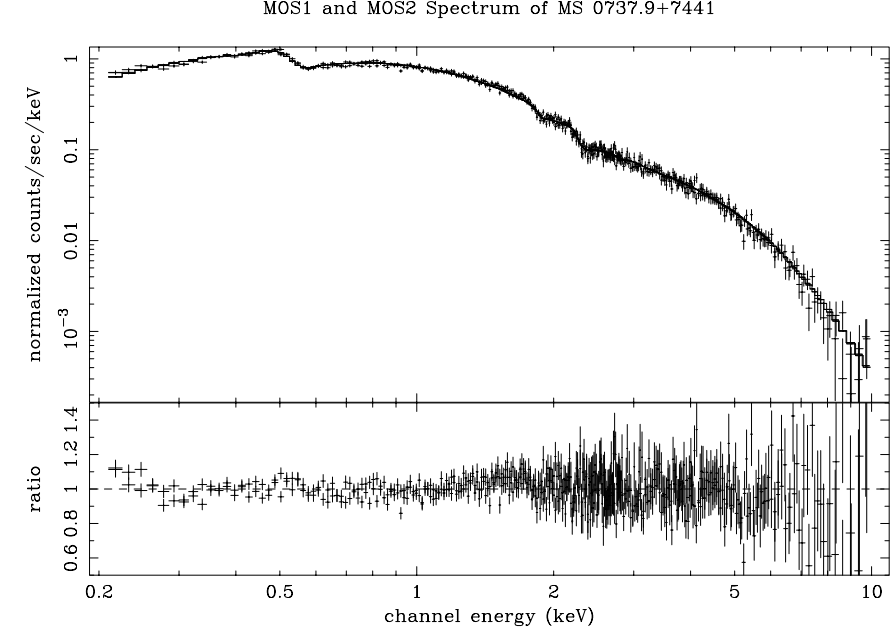

Fig. 2. Power-law fit to the EPIC MOS 1 and MOS 2 data. The upper panel shows the count rate distribution of MS 0737.9+7441 (marked by the crosses) in the EPIC MOS 1 and MOS 2 detectors versus energy. The solid line gives the model spectrum. The lower panel gives the ratio between the data and the model

\subsection{EPIC MOS results}

In the following the spectral fitting results to the combined MOS 1 and MOS 2 observations are presented (cf. Fig. 2). All events within the MOS X-ray pattern library were used (i.e. Patterns 0 to 12). As with the pn, the MOS data in the 0.2 to $10 \mathrm{keV}$ band are well-fit by a single power law with neutral absorption somewhat higher than the nominal Galactic value. A power-law model with the Galactic absorbing column fixed and adding a redshift corrected neutral hydrogen absorbing column density component gives, $N_{\mathrm{H} \text {,fit }}^{z=0.315}=(3.25 \pm 0.25) 10^{20} \mathrm{~cm}^{-2}$ and $\Gamma=2.28 \pm 0.01$. The errors are $90 \%$ confidence for 1 interesting parameter. The reduced $\chi^{2}$ value is 1.27 for 531 d.o.f. No significant systematic difference was found fitting the data from the MOS cameras separately. The residuals to the MOS fit reveal correlated variations which are at most discrepant by $10 \%$ and in general near to the major instrumental absorption edges at carbon, oxygen and silicon. The measured flux is consistent within $5 \%$ of the pn value.

\section{Timing properties}

In Fig. 3 we show the soft $(0.1-1 \mathrm{keV})$ and hard (1-10 keV) light curves for the three continuous observation segments on MS $0737.9+7441$. The amplitude variability does not exceed a factor of about $10 \%$, both in the soft and the hard band. None of the X-ray variability events shown in Fig. 3 exceed the radiative efficiency limit (Fabian 1979; Brandt et al. 1999), sometimes observed in BL Lac objects.

\section{Search for spectral variability}

While X-ray amplitude variations of about $10 \%$ are present in the EPIC light curves, these flux variations are 

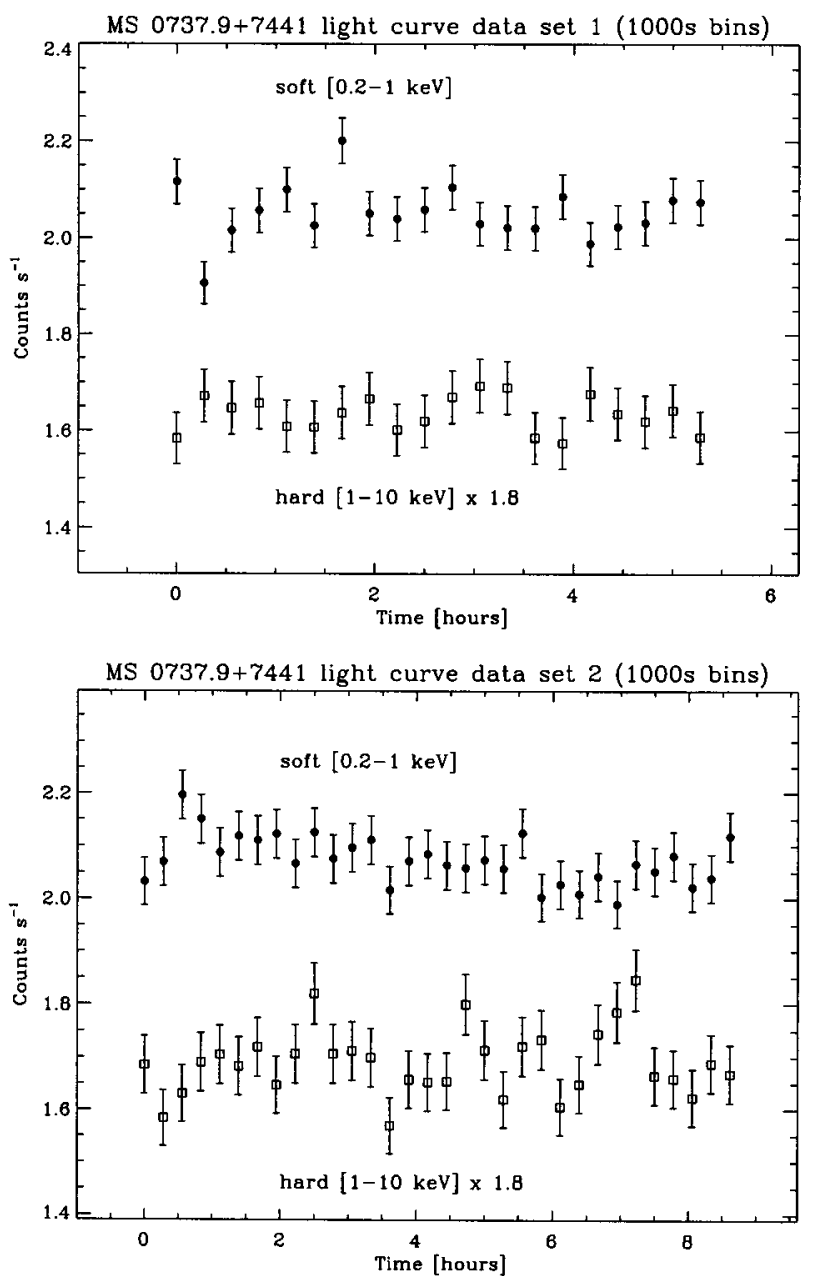

MS 0737.9+7441 light curve data set $3(1000$ s bins)

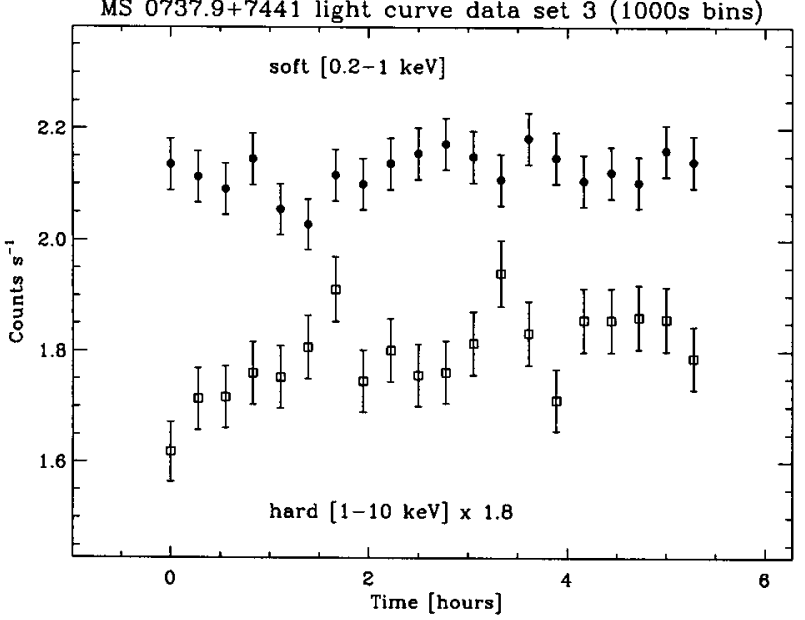

Fig. 3. EPIC pn light curve for the three continuous observation segments in different energy bands

not correlated with significant spectral variability. No significant difference was found in the variability behavior as seen by the pn and MOS cameras, so for brevity we present the pn results only. In Fig. 4 we plot the hardness ratio obtained from the soft $(0.2-1 \mathrm{keV})$ and hard $(1-10 \mathrm{keV})$ energy band in the pn camera versus the count rate. The hardness ratio remains constant within the errors.

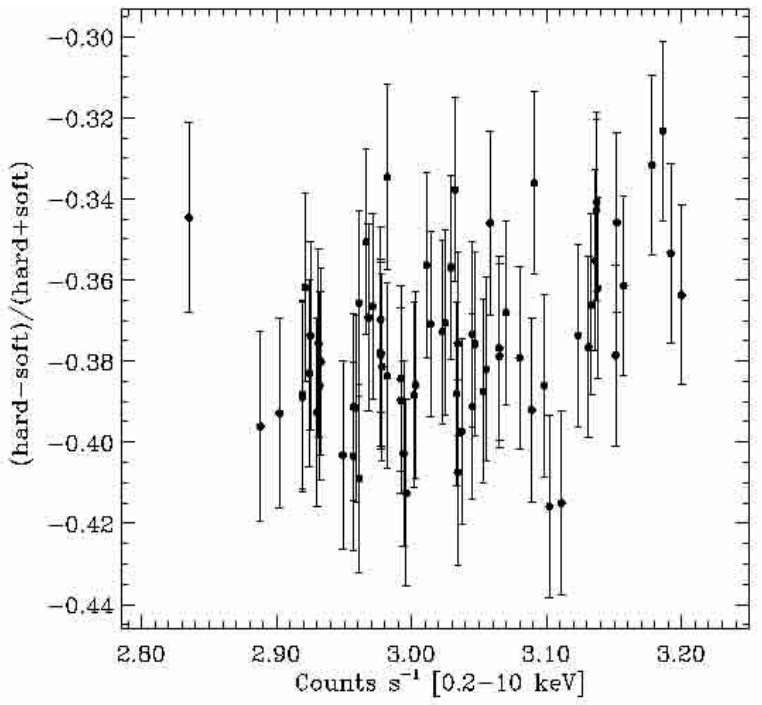

Fig. 4. Hardness ratio obtained from the soft $(0.2-1.0 \mathrm{keV})$ and the hard (1.0-10 keV) energy band ratio versus count rate. No significant spectral variability is detected

\section{Summary}

$X M M-N e w t o n$ observations reveal that a featureless simple power law model with absorption by neutral hydrogen provides an adequate fit to the data. The photon indices as measured with the EPIC pn and EPIC MOS are slightly different with $2.38 \pm 0.01$ and $2.28 \pm 0.01$, respectively, most probably due to present calibration uncertainties for the EPIC detectors. The spectral residua between 0.5 and $0.7 \mathrm{keV}$ might be attributed to uncertainties in the presently available EPIC pn response matrix not allowing us to make definitive statements on the possible presence of soft X-ray emission lines, e.g. emission due to O VIII $(653 \mathrm{eV})$ and Fe XXVII $(726 \mathrm{eV})$ would fall in this energy range. We confirm the presence of intrinsic absorption in the source, which is about $N_{\mathrm{H}, \mathrm{fit}}=3.010^{20} \mathrm{~cm}^{-2}$ in the source frame $\left((2.70 \pm 0.20) 10^{20} \mathrm{~cm}^{-2}\right.$ for EPIC pn and $(3.25 \pm 0.25) 10^{20} \mathrm{~cm}^{-2}$ for EPIC MOS). The soft X-ray absorption detected in MS $0737.9+7441$ appears to be fairly similar to other studies. Beckmann \& Wolter (2000) found a mean value for the intrinsic absorption at soft X-ray energies of about $N_{\mathrm{H} \text {,fit }}=1.010^{20} \mathrm{~cm}^{2}$ with the largest value of $1.010^{21} \mathrm{~cm}^{-2}$. The intrinsic absorption found in the host galaxy of the BL Lac MS 0737.9+7441 is also similar to the soft X-ray absorption of radio-quiet active galaxies (cf. Table 1 of Boller et al. 1996 and Table 2 of Walter \& Fink 1993). The flux variations of about $10 \%$ are relatively small for a BL Lac object (compare Giommi et al. 1990). No significant spectral variations are detected during the observations.

Acknowledgements. It is a pleasure to acknowledge the efforts of the SOC and SSC teams in making the observations possible and for developing the SAS software package used to reduce the 
data. We thank the referee, Eric Perlman, for very useful comments which helped us to improve the paper substantially. The $X M M$-Newton project is supported by the Bundesministerium für Bildung und Forschung/Deutsches Zentrum für Luft- und Raumfahrt (BMBF/DLR), the Max-Planck Society and the Heidenhain-Stiftung. R.G.G and S.S. acknowledge the support of PPARC, United Kingdom and S.V. acknowledges the support of ASI, Italy.

\section{References}

Beckmann, V., \& Wolter, A. 2000 [astro-ph/0007089]

Bevington, P. R., \& Robinson, D. K. 1992, Data Reduction and Error Analysis for the Physical Sciences, Second Edition (McGraw-Hill, New York)

Boller, Th., Brandt, W. N., \& Fink, H. 1996, A\&A, 305, 53
Brandt, W. N., Boller, Th., Fabian, A. C., \& Ruszkowski, M. 1999, MNRAS, 303, L53

Fabian, A. C. 1979, Proc. Roy. Soc. London A, 366, 449

Gioia, I., Maccacaro, T., Schild, R. E., et al. 1990, ApJS, 72, 567

Giommi, P., Barr, P., Garilli, B., Maccagni, D., \& Pollock, A. M. T. 1990, ApJ, 356, 432

Lamer, G., Brunner, H., \& Staubert, R. 1996, A\&A, 311, 384

Morris, S. L., Stocke, J. T., Gioia, I. M., et al. 1991, ApJ, 380, 49

Perlman, E. S., Stocke, J. T., \& Wang, Q. D. 1996, ApJ, 456, 451

Stocke, J. T., Morris, S. L., Gioia, I. M., et al. 1991, ApJS, 76, 813

Walter, R., \& Fink, H. 1993, A\&A, 274, 105

Wolter, A., Comastri, A., Ghisellini, G., et al. 1998, A\&A, 353, 899 